\title{
Surgical treatment of preschool childhood spinal thoracic tuberculosis: two case reports and technical note
}




\title{
Surgical treatment of preschool childhood spinal thoracic tuberculosis: two case reports and technical note
}

\author{
Mehmet Nuri Erdem ${ }^{1}$, Anas Abdallah'², Sinan Karaca ${ }^{3}$, Mehmet Aydoğan $^{4}$ \\ ${ }^{1}$ Department of Orthopaedic Surgery, Hisar Intercontinental Hospital, Umraniye/Istanbul, \\ TURKEY \\ ${ }^{2}$ Department of Neurosurgery, Bezmialem Vakif University, Istanbul, TURKEY \\ ${ }^{3}$ Department of Orthopaedic Surgery, Fatih Sultan Mehmet Training and Research Hospital \\ Atasehir, Istanbul, TURKEY \\ ${ }^{4}$ Orthopaedist, Fulya Orthopaedic and Spine Institute, Istanbul, TURKEY
}

\begin{abstract}
Background: The development of paraplegia and kyphosis are two important problems related to spinal tuberculosis (Pott's disease) in children. The current study aims to present two pediatric Pott's disease, point out the progression of this disease in children, surgical options and surgical outcomes. Materials and methods: Two cases of childhood Pott's disease were referred to our department with progressive neurological deficits (both of children were experienced bilateral weakness in lower extremities; grade $\mathrm{C}$ according to Frankel scale) during short periods (less than one month per each). Both cases' MRIs are demonstrated abscesses in thoracic spine. Both patients are treated surgically. We discussed in this study the technical notes in both cases, especially in 2year-old child. Results: Both cases were treated surgically after decompressing nerve roots and thecal sac posterior instrumentation was applied. Microbiological examination of both samples revealed on M.Tuberculosis. Orthoses are applied three months after operations. Antituberculosis medication protocol was applied. Preoperative paraparesis had recovered completely and muscle strengths all returned to normal in both children. There is no loss of correction during the follow-up periods. Conclusion: Spinal tuberculosis is a dynamic disease that may lead to severe deformities in childhood. Especially in cases where two or more vertebrae are affected, early surgical treatment is essential to prevent severe kyphosis. Surgical intervention accompanied with antituberculosis medications protocol is essential to cure treatment in pediatric population. In children aged $\leq 6$ years and more than one vertebrae were affected, fibula can be used to allow growth of the child vertebral column.
\end{abstract}

Key words: Childhood Pott's disease, corpectomy, instrumentation, spinal tuberculosis, fibula as autograft 


\section{Introduction}

Spinal tuberculosis (Pott's disease), is the most common form of extrapulmonary tuberculosis. The incidence of this condition has shown a rapid increase recently in especially undeveloped or developing countries [1]. Unfortunately, spinal tuberculosis in children is different from their adult counterpartners. In the treatment of the child spinal tuberculosis, we have to find answers to two major questions; first, what is the clinical presentation of child patient who was involved with Pott's disease? Second, does medical treatment sufficient to treat child patient? Recent technological advancements in diagnosis of spinal tuberculosis in adults have not been validated in children. For example, MRI scanning in preschool children needs general anesthesia. Similarly, trials of new medications and development of pediatric formulations of standard first- and second-line medications are lagging behind [2]. Although the condition can be successfully treated with antituberculosis medications, the development of paraplegia, kyphosis and even sharp angular kyphosis (gibbous) deformity are significant problems. Especially childhood spinal tuberculosis with destruction of two or more thoracic vertebrae may result in severe spinal tuberculosis and a progressive kyphosis deformity that exceeds 100 degrees may occur. These deformities are often associated with neurologic deficits and respiratory problems [3]. In such cases, medical treatment will not be sufficinet to correct such deficits and deformities without surgery.

In the present two reports, the authors aim to present the surgical techniques and outcomes of Pott's diseases in preschool pediatric population. Pott's disease acts progressively in preschool children, however, surgical intervention combined with antituberculosis medications gives good results.

\section{Case reports}

\section{First case report}

A 4-year-old boy was referred to our department with back pain and failure to walk that began one month ago and progressed thereafter. He had severe spastic paraparesis and his neurologic condition was assessed as grade $\mathrm{C}$ according to the Frankel scoring system. MRI showed destruction at T 4-5-6 levels, proximal thoracic gibbous deformity, and that the local kyphosis angle between T3T7 had increased to 71 degrees (Figure $1 \mathrm{~A}, \mathrm{~B}$, C). A paraspinal abscess that caused severe anterior compression at T4-5-6 levels and compression of the spinal cord due to destruction in the vertebrae were detected. Destruction of discs and bones was notably. Therefore, presumptive diagnosis was that of Pott's disease.

\section{Surgical Procedure}

Under general anesthesia and using intraoperative neurophysiological monitoring (IONM) the patient is positioned prone using supporting roll on each side to preserve the current kyphosis. A midline skin incision is performed between spinous processes of $\mathrm{T} 1$ and T9. After dissecting the paraspinal muscles which are retracted laterally. The laminae and transverse processes between $\mathrm{T} 2$ T8 are exposed. Bilateral pedicle screws were inserted at T2-3 proximally, and T7-8 distally, then an appropriately shaped temporary rod was placed to the right and locked (Stryker 
XIA pediatrics). Total posterior laminectomies are performed at levels T4-T5T6. The abscess drainage is done upon opening of the posterior lamina. The dissection is advanced bilaterally at levels T4T5-T6, exposing the costotransverse joints and the proximal aspects of the ribs. After the pleura is carefully stripped off the vertebrae, the heads of the 4 th, 5th, and 6 th ribs are bilaterally excised together with costotransverse joints Paraspinal dissection is completed by bilateral ligation of T4-5-6 nerve roots. The dissection is advanced retropleurally, between the vertebra and pleura. First the T3-T4 disc, followed by the T6-T7 disc, are evacuated and the disc spaces are cleaned with osteotomes and curettes. The vertebral bodies of T4-5-6 are removed together with sequestered bone fragments.
The surgical field is irrigated with copious saline. Dura decompression is performed fully, thus spinal cord compression is removed. The closing-opening wedge osteotomy, described by Rajasekaran et al. [3], is performed and correction is achieved without traction or folding of the spinal cord. Rods contoured according to physiologic kyphosis are implanted. Then a mesh cage, filled with allogenous graft, is placed between corpuses of T3 and T7. This followed by the posterior compression (Figure 2A, 2B). The correction is evaluated using fluoroscopy, then the system is locked over the rods. Especially during the correction stage, the neurologic condition is controlled using IONM through the operation. No neurologic complications were recorded during or after the operation.

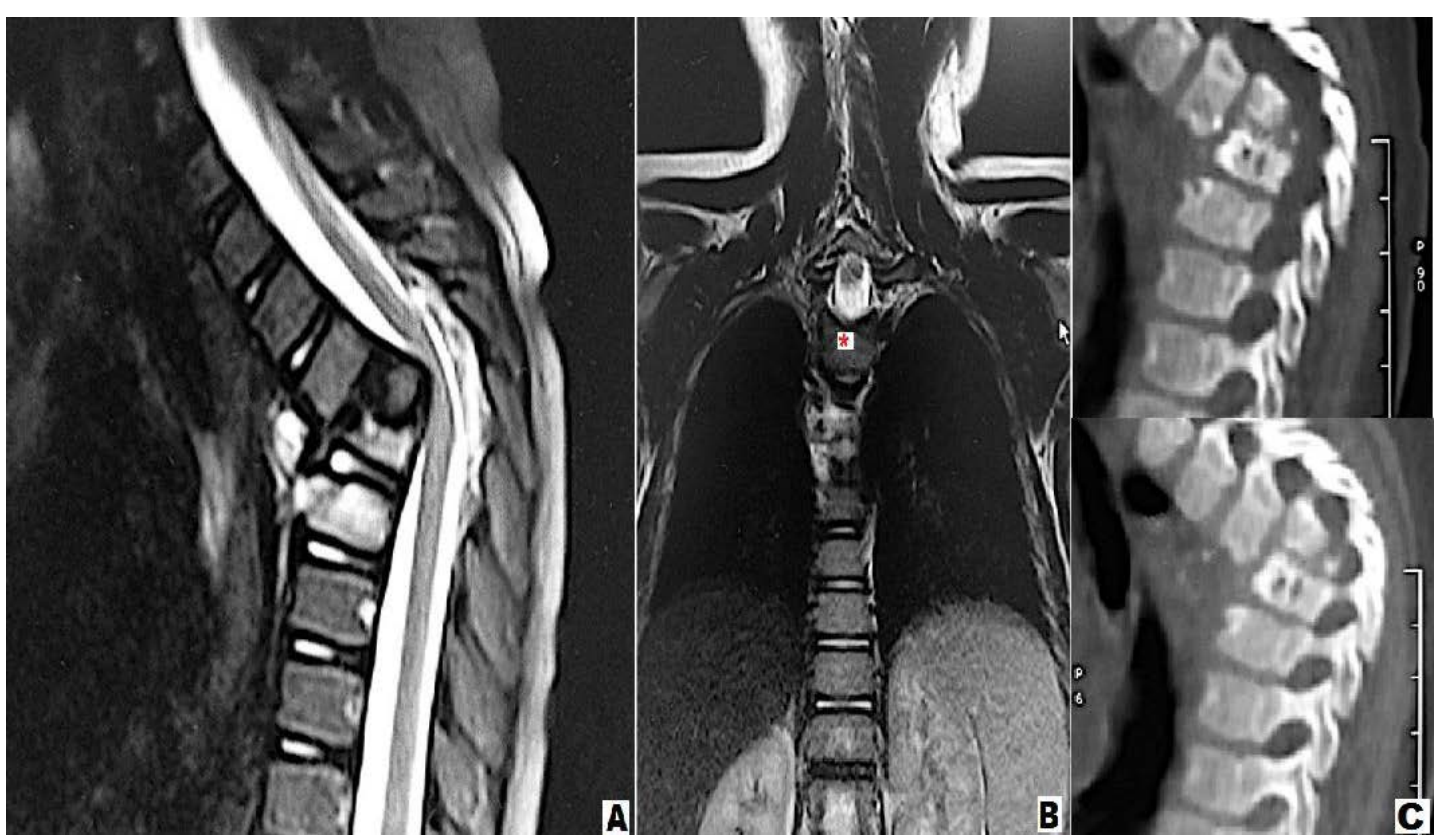

Figure 1 - 1.Case's preoperative MRI demonstrated a large paraspinal abscess and kyphosis. [A]: Sagittal T2weighted image; [B]: Coronal T2-weighted image: abscess formalation was indicated by $\left({ }^{\star}\right)$; $[\mathrm{C}]$ : Preoperative CT scan images 

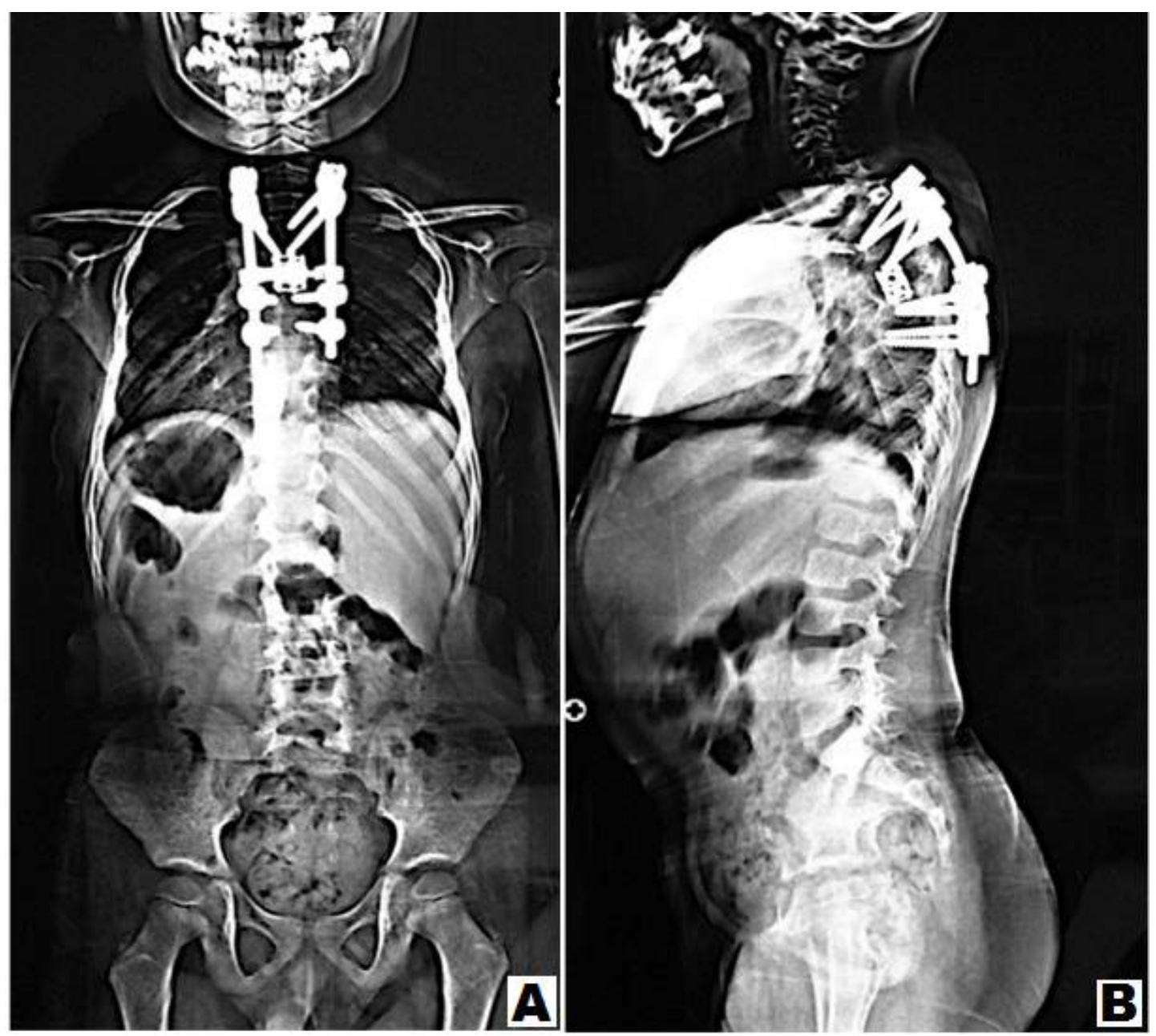

Figure 2 - 1.Case's Early Postoperative radiographic images. [A]: Lateral plain; [B]: AP plain

\section{Second case report}

A 2-year-old boy presented with severe back pain and paraparesis of one-month duration. Neurological examination revealed a paraparesis, which was evaluated as grade $\mathrm{C}$ according to Frankel Scoring System. Thoracic MRI demonstrated destruction with a large paraspinal abscess involving T5-T6-T7 levels, compressing the spinal cord (Figure 3) [4]. Destruction of discs and bones at the same time was notably. Therefore, presumptive diagnosis was that of Pott's disease. X-rays of chest and thoracic spine showed destruction at the T5-T6-T7 levels and the local kyphosis angle was $37^{\circ}$ at this level (Figure 4B). There was no other infection focus in physical examination, radiological, hematological and biochemical tests. Emerge surgical procedure was performed after preoperative evaluation was completed. 
Surgical Procedure

Under general anesthesia using transthoracic approach after removing the fifth rib in right side and IONM the patient is positioned lateral decubit position. After exposure, the paraspinal abscess drained spontaneously. T5, 6 and T7 corpectomies are performed. Decompression was carried out until duramater was visible through anterior approach, so all affected vertebrae are extracted. Anterior fusion is performed with structural one autogenous fibula and one autogenous rib graft. Periost of the fibula is prevented and closed anatomically after resection. Anterior instrumentation using one screw to each vertebra at $\mathrm{T} 4$ and $\mathrm{T} 8$ is performed. The system is locked, thorax drainage system is applied and thoracotomy is closed. The patient is positioned prone. Bilateral polyaxial pedicle screws are inserted at T4 and T8 levels and rods are placed for permanent fixation. Pediatric spinal screw systems are preferred for the immature pedicles (Figure 4A, 4C, 4D).
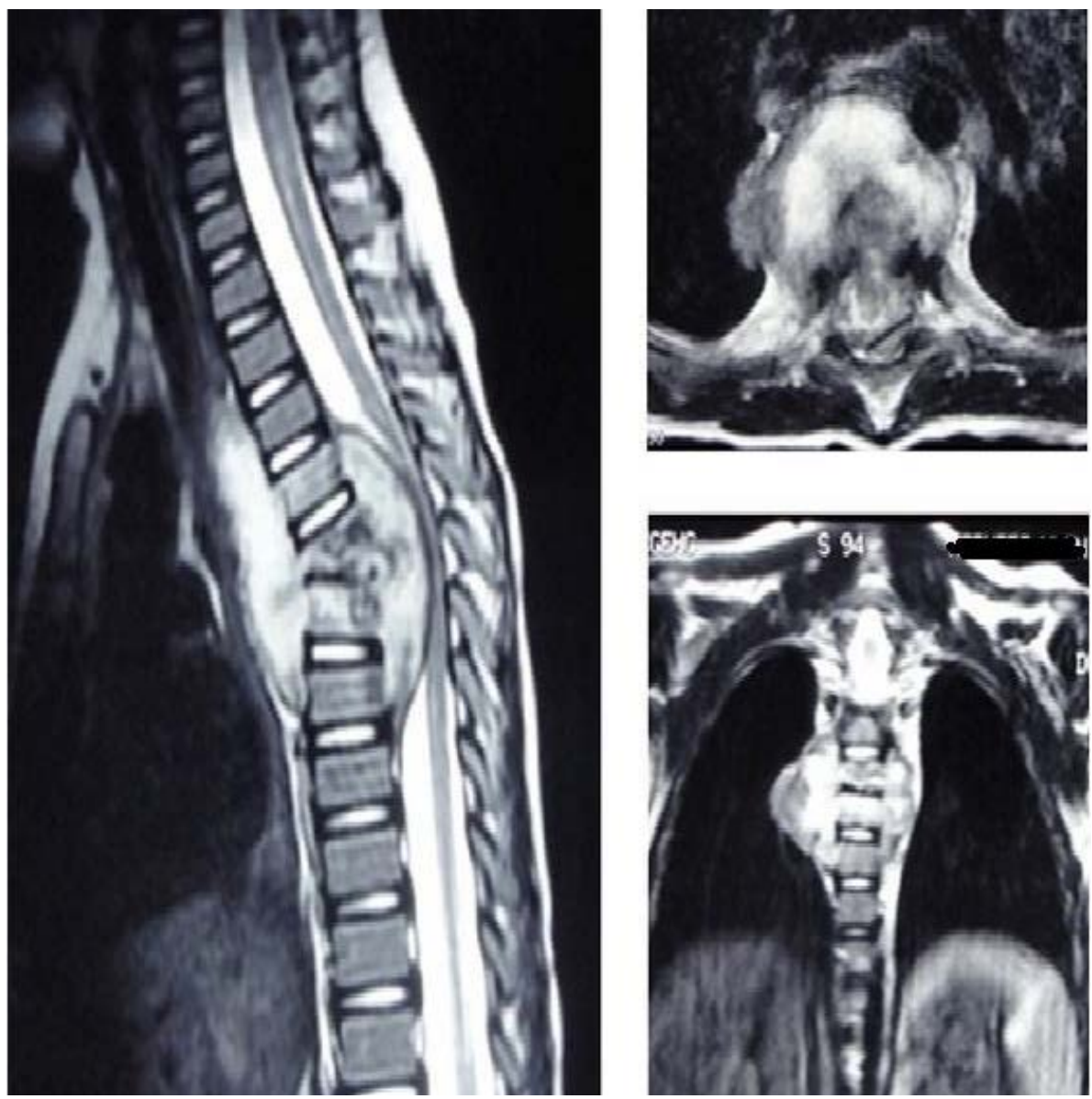

Figure 3 - 2. Case's preoperative MRI; sagittal, axial and coronal T2-weighted images 


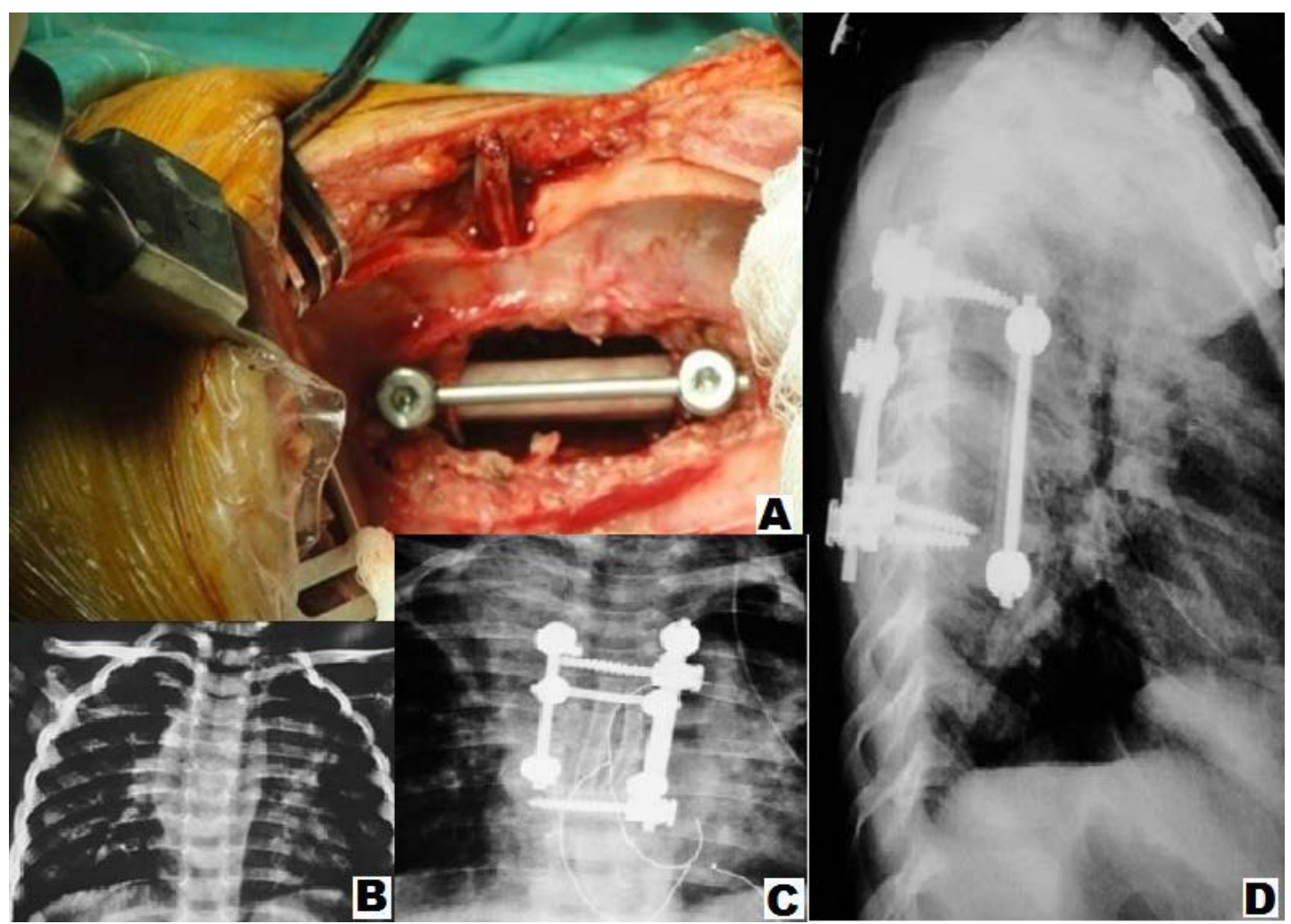

Figure 4 - 2. Case's intraoperative and radiographic images. [A]: Intraoperative photo shows anterior instrumentation with structural autogenous fibula and autogenous rib grafts; [B]: Preoperative AP plain X-ray;

$[\mathrm{C}]$ : Postoperative sixth week AP plain x-ray; [D]: Postoperative sixth week lateral x-ray

\section{Postoperative period}

Microbiological examination of both samples which were obtained from the abscesses and soft tissues revealed on Mycobacterium Tuberculosis. A Minerva orthosis in first case and thoracolumbosacral orthoses in second case are applied three months after operations. Antituberculosis treatment protocol was applied for nine months (INH-rifampicin-pyrazinamidestreoptomycin for 3 months, followed by INHrifampicin-pyrazinamide). The patients were discharged five and eight days after the operations, respectively. 1. Case could walk inside the room with the help of an orthosis. 2. Case could sit on the bed or walk around one week after surgery. On their 6th week, preoperative paraparesis had recovered completely, muscle strengths all returned to normal and the neurologic condition was evaluated as Frankel E in both children. On their 6th, 12th, 24th and 36th (for second case) month follow-up visits, local kyphosis angle between both of T3 and T7 measures 31 and 32 degrees, respectively. There is no loss of correction during the follow-up period. 


\section{Discussion}

Vertebral tuberculosis is an instable lesion that progresses until bone fusion, and the best proof that it has healed is formation of fusion [5]. In general, kyphosis is detected in more than $80 \%$ of the patients at the time of referral [6]. The disease is cured with antituberculosis drugs, however the deformity persists. In cases with severe bone destruction, the healthy vertebra moves forward and makes contact with the distal healthy vertebra. These patients (3-5\%) end up with greater than 60 degrees deformity [7]. The final outcome of the deformity depends on the initial vertebral destruction, patient's age and the level of the lesion [8].

The Pott's disease is relatively rare diagnosed in preschool children. Recently published study reported 43 cases of Pott's disease which were diagnosed in patients aged between 10-69 years [9]. Despite the fact that the study was carried out in Younde which is considered as one of the endemic regions of Pott's disease, the study did not report any preschool Pott's patient. The pediatric spine is more flexible and prone to destruction. Unfortunately, if the deformity at the time of diagnosis is greater, it has a greater tendency for collapse during recovery [8]. Additionally, the progression of the deformity continues once the disease has healed, because of continued skeletal growth [3]. In preschool pediatric patients ( $<6$ years), the correction surgery still a challenge for spine surgeons. In case 1 , we could have avoided cutting nerve roots on one side as we need to more space on the side from where we want to insert the cage, while on the other side, only debridement is needed that can be done without cutting the nerve roots. We could have used fibula in this case too as fibula has the tendency to increase its diameter with growth. Case 2 is only 2 years old, without much kyphotic deformity. We could have just done costotransversectomy, draining the abscess and posterior fusion at that level to avoid crankshaft phenomenon in future, followed by chemotherapy.

To understand those spinal tuberculosis cases under a risk of progressive deformity, Rajasekaran et al. tried to determine some of the factors during the early phase of the disease. According to their study, facet joint separation; posterior retropulsion of the affected vertebral segment; lateral translation of the vertebral column; and anterior contact of the upper and lower healthy vertebra due to anterior movement of the upper healthy vertebra are defined as risk factors, and the authors emphasized the signficance of achieving bone fusion during the early phase by treatment [10].

Numerous options have been defined for the surgical treatment of spinal tuberculosis, including anterior debridement and arthrodesis [11], plates and screws [12, 13], titanium cages [14], and posterior instrumentation combined with single or two staged anterior decompression $[15,16]$. Fusion with anterior debridement and autogenous bone grafts are accepted as the classical method. It provides effective debridement, a rapid bone union occurs with grafts, also the kyphosis and progressive collapse are prevented [17]. However in cases where two or more levels are affected, it is 
more difficult to obtain successful results with this method because of graft failure and progressive kyphosis deformity $[1,12,18]$. Anterior radical debridement and/or posterior instrumentation are necessary to preserve the achieved correction $[18,19]$. According to our experience, in spinal tuberculosis cases with severe kyphosis where 2 or more areas are affected, titanium cage and $360^{\circ}$ with posterior instrumentation method provides effective stabilization, early mobilization, effective correction of the kyphosis and maintaning the correction after the operation [15].

Despite that the fact appropriate antituberculosis therapy is still the basis of treatment of tuberculosis in general and of Pott's disease in particular, surgerical intervention still plays a diagnostic and therapeutic role especially in cases which patients referred at a late stage after complications have developed (spinal compression, deformity and instability). Presence with severe neurological deficit at surgery is an important prognostic factor. Surgical decompression should be early as soon as possible before a total neurological deficit is developed, which signifies the presence of irreversible, ischemic lesions (Frankel A) [9]. In patients with sharp angle gibbosity having also severe bone destruction, corpectomy with the posterior approach to avoid the morbidity of decompression through the anterior route, evacuation of the area anterior to the spinal cord, and gradual correction of local kyphosis with closingopening wedge osteotomy is an effective and reliable method [2]. In order to increase the stability of the cage placed into the corpectomy area and maintain the correction achieved, a posterior instrumentation was also performed. In a study which discussed the safety of transpedicular instrumentation was the best method for achieving a stable fixation in children [20].

The major limitation of this study was presence of two preschool pediatric Pott's disease which were analyzed retrospectively. Randomized prospective controlled studies with larger series and a longer follow-up period are required in the future.

\section{Conclusion}

In cases of thoracic vertebral osteomyelitis with severe anterior column destruction in the toddlerhood (preschool period) age, the use of anterior structural grafts and instrumentation in combination with posterior instrumentation is safe and effective in maintenance of the correction achieved and allows efficient stabilization and early mobilization. However, thoracic spinal tuberculosis cases cause severe anterior column destruction in preschool children, posterior instrumentation, corpectomy and closing-opening wedge osteotomy approach is enough.

\section{Correspondence}

Anas Abdallah, MD

Department of Neurosurgery, Bezmialem Vakif University, Adnan Menderes Bulvari, Vatan Street, 34093 Fatih, Istanbul, Turkey

E-mail:abdallahanas@hotmail.com; aabdallah@bezmialem.edu.tr

Phone: +90 212 5232288-2175 Cell-Phone: +905532233535Fax: +902124531869 


\section{References}

1.Moon MS: Spine update: tuberculosis of the spine. Spine 1997; 22: 1791-1797.

2.Swaminathan S, Rekha B: Pediatric tuberculosis: global overview and challenges. Clinical Infectious Disease 2010; 50(53): S184-S194.

3.Rajasekaran S, Kanna PRM, Shetty AP: Closing Opening wedge osteotomy for severe, rigid, thoracolumbar post - tubercular kyphosis. Eur Spine J 2011; 20: 343-348.

4.Erdem MN, Sever C, Korkmaz MF, Karaca S, Kirac F, Tezer M: Pott's disease in 2-year-old child treated by decompression and anterior-posterior instrimented fusion (Case report): Case Report in Orthopedics 2014; 2014: Article ID 252973.

5.Boachie-Adjei O, Squillante RG: Tuberculosis of the spine. Orthop Clin North Am 1996; 27: 95-103.

6.Moon MS, Lee MK: The changes of the kyphosis of the tuberculous spine in children following ambulant treatment. Korean Orthop Assoc 1971; 6: 203-208.

7.Yilmaz C, Selek HY, Gurkan I, Erdemli B, Korkusuz Z: Anterior instrumentation for the treatment of spinal tuberculosis. J Bone Joint Surg (Am) 1997; 81: 1261-1267. 8.Rajasekaran S: The problem of deformity in spinal tuberculosis. Clin Orthop 2002; 398: 85-92.

9.Djientcheu VP, Mouafo Tambo FF, Ndougsa IS, Eloundou NJ, Kouna Tsala IN, Ngowe Ngowe M, Andze OG, Sosso MA: The role of surgery in the management of Pott's disease in Younde. A review of 43 cases. Orthopaedics and Traumatology: Surgery Et Research 2013; 99? 419-423.

10.Rajasekaran S: The natural history of childhood spinal tuberculosis: A 15-year prospective study. J Bone Joint Surg Br 2001; 83: 954-962.

11.Medical Research Council Working Party on Tuberculosis of the Spine: A 10-year assessments of controlled trials comparing debridement and anterior spinal fusion in the management of tuberculosis of the spine in patients on standard chemotherapy in HongKong. J Bone Jt Surg (Br) 1982; 64: 393-398.

12.Benli IT, Acaroğlu E, Akalın S, Kis M, Duman E, Un A: Anterior radical debritment and anterior instrumentation in tuberculosis spondylitis. Eur Spine J 2003; 12: 224-234.

13.Ruf M, Harms J: Pedicle Screws in 1- and 2-Year-Old Children: Technique, Complications, and Effect on Further Growth. Spine 2002; 27: 460-466.

14. Hee H, Majd M, Holt R, Pienkowski D: Better treatment of vertebral osteomyelitis using posterior stabilisation and titanium mesh cages. J Spinal Disord 2002; 15: 149-156.

15.Erturer E, Tezer M, Aydogan M, Mirzanli C, Ozturk I: The results of simultaneous posterior-anterior-posterior surgery in multilevel tuberculosis spondylitis associated with severe kyphosis. Eur Spine J 2010; 19: 2209-2215.

16.Moon MS, Woo YK, Lee KS, Kim SS, Sun DH: Posterior instrumentation and anterior interbody fusion for tuberculous kyphosis of dorsal and lumbar spine. Spine 1995; 20: 1910-1916.

17.Hodgson AR, Stock FE: Anterior spinal fusion: Preliminary communication on radical treatment of Pott's disease and Pott's paraplegia. Clin Orthop Relat Res 1994; 300: 16-23.

18.Kim DJ, Yun YH, Moon SH, Riew KD: Posterior instrumentation using compressive laminar hooks and anterior interbody arthrodesis for the treatment of tuberculosis of the lower lumbar spine. Spine 2004; 29 : 275-279.

19.Fukuta S, Miyamoto K, Masuda T, Hosoe H, Kodama $\mathrm{H}$, Nishimoto H, Sakaeda H, Shimizu K: Two-stage (posterior and anterior) surgical treatment using posterior spinal instrumentation for pyogenic and tuberculotic spondylitis. Spine 2003; 28: 302-308.

20.Tuli SM: Severe kyphotic deformity in tuberculosis of the spine. Int Orthop 1995; 19: 327-333. 